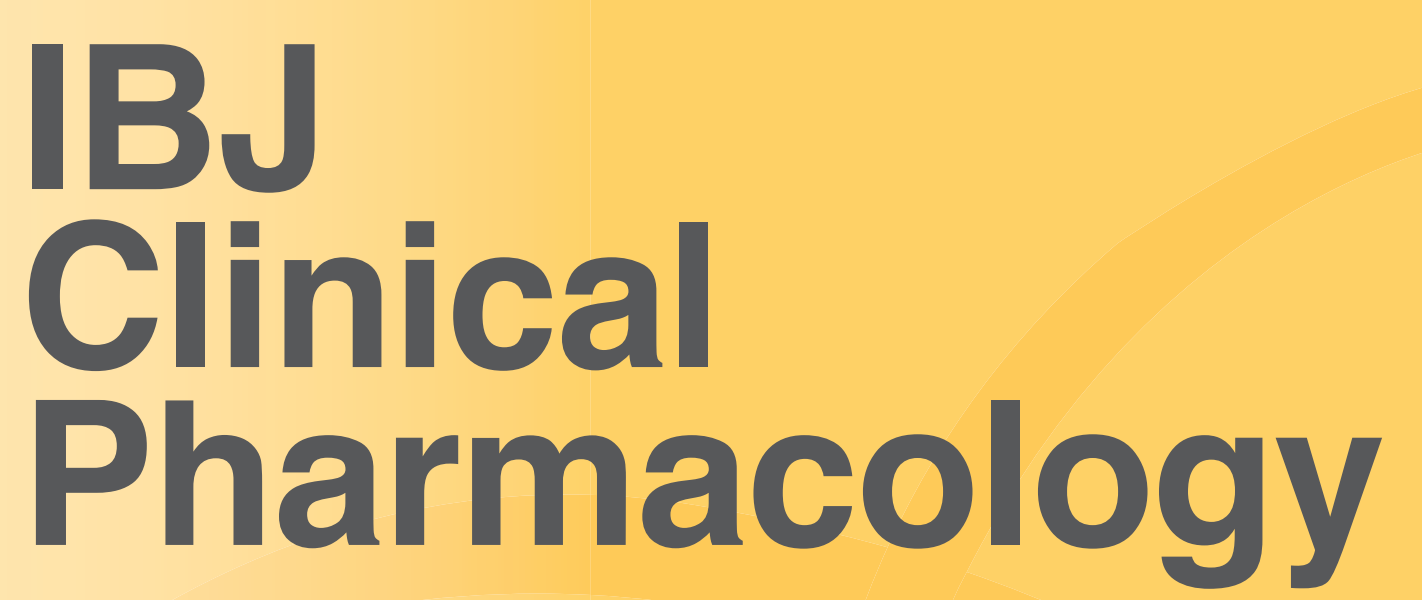

Volumen 1, Suplemento Especial 1, Noviembre 2017

\title{
PIELen Red
}

Abstracts de las IV Jornadas de PIELenRED.

Madrid, 20 de octubre de 2017 


\title{
The experience of the patient with epidermal necrolysis (TE) in the Getafe Hospital's Great Burned Unit (GBU) from their own perspective.
}

\author{
Sandra Fernández Núñez ${ }^{1}$,Natalia Cámara Conde², Amelia Ymelda Moscoso González ${ }^{3}$. \\ ${ }^{1}$ DUE Unidad Grandes Quemados, Hospital Universitario Getafe, España. Email: sfernandezn@salud.madrid.org \\ ${ }^{2}$ DUE Unidad Grandes Quemados, Hospital Universitario Getafe, España. Email: Natalia.camara@salud.madrid.org \\ ${ }^{3}$ DUE Unidad Grandes Quemados, Hospital Universitario Getafe, España. Email: aimg2486@hotmail.com
}

Introduction: NE is a disease characterized by a detachment of the epidermis and mucous membranes that requires specialized care in GBU. The objective of this study is to know the experience lived by patients admitted to our unit to implement improvements that provide a less traumatic experience and with fewer sequelae to future patients.

Material and method: A self-designed questionnaire was used through telephone interviews, consent was verbally given. All patients admitted since $2014(n=8)$ were selected, one declined. The results were obtained after an exhaustive analysis of the answers of the questionnaire until saturation of the discourse.

Results: All patients overall value their stay in the unit as very satisfactory. The best value has been the treatment received, the professionalism of the staff and the personal care. After numerous previous misjudgments, being in a place where staff have experience about the process gives them security. The most negative assessment corresponds to the adequacy of the facilities, especially in the passage to regular care unit.

The knowledge of the patient's name by the staff and vice versa has been a highly valued aspect.

As for the physical environment, the majority $(n=6)$ was considered adequate, with nocturnal noise being the most annoying.

Patients with great affection of the oral mucosa had the need to personalize the diet.

It was positively valued that they were encouraged to collaborate in their self-care, although they had appreciated certain measures aimed at protecting their privacy.

Patients have considered as good or very good pain management, poor quality of their rest and very positive flexibility in visiting standards.

The majority $(n=5)$ reported having lived with calm the transfer to regular care unit and discharge to home.

The overall perception of their experience is equally divided between very positive and very negative; All the patients experimented fear, loneliness $(n=3)$, isolation $(n=4)$ and other emotions such as worry, helplessness and insecurity.

At discharge, a few $(n=2)$ reported having affected basic activities of daily living, while half referred affectation of their previous activities and a large majority $(n=5)$ their working life, leisure and social and sexual relationships. The most important sequel is the ocular affectation.

Aspects that would have significantly improved their experience are: night noise reduction, more personalized attention, longer visit time and follow-up after discharge.

Conclusions: from all of the above, it is possible to implement measures that are easy and cheap to apply that would improve the experience of patients with NE in our unit.

Keywords: patient, TEN, Great Burned Unit, vital experience. sequelae.

Published November 9, 2017.

Copyright: (C) 2017 Author. This is an open-access article distributed under the terms of the Creative Commons Attribution License, which permits unrestricted use, distribution, and reproduction in any medium, provided the original author and source are credited. Editor: Alberto M. Borobia Pérez.

Cite as: Fernández Núñez S.,Cámara Conde N., Moscoso González AY. The experience of the patient with epidermal necrolysis (TE) in the Getafe Hospital's Great Burned Unit (GBU) from their own perspective. IBJ Clin Pharmacol 2017 (S1):e0001 doi: 10.24217/25304984.17v1s1.00001.

Funding: The authors declare no funding involved.

Competing Interests: The authors declare no conflicts of interest. 


\title{
Tratamiento oftalmológico agudo del paciente con necrolisis epidérmica tóxica.
}

\author{
M.Y. González Ruiz¹
}

${ }^{1}$ Servicio de Oftalmología, Hospital universitario de Getafe, Madrid, España E-mail: ygonzalez.hugf@salud.madrid.org.

Introducción: La necrólisis epidérmica tóxica (NET) o Síndrome de Lyell es una enfermedad inmunológica que se caracteriza por una afectación dermatobullosa y erosiones de las membranas mucosas, con alta morbilidad y mortalidad. Se trata de una reacción de hipersensibilidad retardada precipitada por agentes víricos, bacterianos, farmacológicos o neoplásicos. La superficie ocular es una de sus principales dianas y puede ocasionar una ceguera irreversible a pesar de recibir cuidados exhaustivos en unidades especializadas.

Material y métodos: Con este artículo se pretende exponer el protocolo de tratamiento oftalmológico en la fase aguda de la NET que aplicamos desde el año 2006, haciendo especial hincapié en el trasplante de membrana amniótica sin suturas que se realiza de forma precoz. Dicho protocolo está basado en los estudios publicados y en la experiencia personal del autor en el manejo de estos pacientes en un centro hospitalario que dispone de Unidad de grandes quemados. La membrana amniótica crio-preservada que se utiliza es procesada en el mismo hospital en parches de $5 \times 5 \mathrm{~cm}$.

Resultados: La severidad de las secuelas cicatriciales conjuntivales, palpebrales y corneales han disminuido mucho en los pacientes a los que se le ha aplicado este tratamiento en relación a los tratados antes del 2006.Debido a esto también se objetiva una mejor función visual.

Conclusiones: El cuidado oftalmológico exhaustivo, junto con el trasplante de membrana amniótica precoz recubriendo toda la superficie ocular en la fase aguda de la NET, es primordial para evitar secuelas graves que incapaciten la vida normal de estos pacientes.

Keywords: toxic epidermal necrólisis, amniotic membrane transplantation, drug-induced disease.

Published November 9, 2017.

Copyright: (C) 2017 Author. This is an open-access article distributed under the terms of the Creative Commons Attribution License, which permits unrestricted use, distribution, and reproduction in any medium, provided the original author and source are credited. Editor: Alberto M. Borobia Pérez.

Cite as: González Ruiz MY. Tratamiento oftalmológico agudo del paciente con necrolisis epidérmica tóxica. IBJ Clin Pharmacol 2017 (S1):e0002 doi: 10.24217/2530-4984.17v1s1.00002.

Funding: The authors declare no funding involved.

Competing Interests: The authors declare no conflicts of interest. 


\title{
Sospecha de NET por Alergical expectorante en ausencia de datos histológicos y microbiológicos.
}

\author{
Díaz García L. ${ }^{1}$, Rodríguez Mariblanca A. ${ }^{1}$, García García I. ${ }^{1}$, Cabañas Moreno MR. ${ }^{2}$, Martínez de Soto L. ${ }^{1}$, Ramírez \\ García E. ${ }^{1}$
}

${ }^{1}$ Servicio de Farmacología Clínica, Hospital Universitario La Paz, IdiPaz, Consorcio PIELenRed, Facultad de Medicina, Universidad Autónoma de Madrid, España ${ }^{2}$ Servicio de Alergia, Hospital Universitario La Paz, IdiPaz, Consorcio PIELenRed, Madrid, España. *Corresponding author: Elena Ramírez. Servicio de Farmacología Clínica, Hospital Universitario La Paz, IdiPaz, Consorcio PIELenRed, Facultad de
Medicina, Universidad Autónoma de Madrid, España, Dirección: Paseo de la Castellana 261. 28046, Madrid, España. Tel - fax: + 34 917277559;
E-mail: elena.ramirez@inv.uam.es

Introducción: La necrólisis epidérmica tóxica (NET) es una enfermedad mucocutánea potencialmente mortal, inducida principalmente por fármacos. Su manifestación consiste de inicio en síntomas gripales (malestar, fiebre y anorexia) en la fase prodrómica, seguido de inflamación cutánea y mucosa (ocular, oral y genital) y dolor; y otras implicaciones sistémicas. El diagnóstico es clinicopatológico, la histopatología confirma el proceso patológico. En la infancia la NET debe diferenciarse fundamentalmente del síndrome estafilocócico de piel escaldada. Además la NET puede estar causada por infecciones (virus Coxsackie A6, virus de la familia herpes, Chlamydia, Mycoplasma, etc).

Material y métodos: Niño de 14 meses que presentó eritema generalizado con despegamiento epidérmico al tacto con afectación 60-70\% de superficie corporal, iniciado en tronco extendido a cara y cuero cabelludo sin afectación ocular ni mucosas y afebril. Se trasladó a nuestro hospital por sospecha de NET, empeoramiento clínico con fiebre, elevación de reactantes de fase aguda, shock séptico, fracaso renal agudo, ascitis, coagulación intravascular diseminada, disfunción cardíaca, trombopenia y anemización. Se realizaron curas intraoperatorias de las lesiones y se realizó tratamiento con hidrocortisona y dexametasona. La evolución posterior fue favorable por lo que fue dado de alta con seguimiento de las lesiones. Durante 5 -7 días previos al inicio del cuadro estuvo en tratamiento con salbutamol, prednisona, dexclorfeniramina y Alergical expectorante ${ }^{\circledR}$ (guaifenesina, diprofilina, paracetamol y clorfenamina) por episodio de broncoespasmo. Fue diagnosticado de causalidad posible por guaifenesina o diprofilina (probable +4 , algoritmo ALDEN, Algorithm of drugs causality for EN) sin poderse descartar una causa infecciosa por clamidia o por virus coxsackie A6 (resultado PCR enterovirus positivo) y en ausencia de biopsia diagnóstica.

Resultados: El SJS/NET es una entidad difícil de diferenciar de otras entidades dermatológicas y/o procesos infecciosos. La infección por Chlamydia pneumoniae y la coinfección por Mycoplasma pneumoniae puede causar SJS/EMM en pacientes con síntomas respiratorios. Otras infecciones víricas como $\mathrm{S}$ mano-boca-pie producida por enterovirus pueden causar trofismo cutáneo y/o de mucosas simulando SJS/NET. Por ello el diagnóstico histológico es necesario. El paciente tomaba guaifenesina del que se ha descrito un caso de reacción anafiláctica y diprofilina (xantina, con efecto similar a la teofilina), habiendo casos descritos de SJS con teofilina.

Conclusiones: En nuestro paciente la ausencia de datos histopatológicos de la enfermedad así como el estudio completo microbiológico impide la confirmación del diagnóstico de SJS/NET, no pudiendo descartar una causa alternativa del cuadro clínico. Asimismo la escasa literatura científica de seguridad de los medicamentos "probables" sospechosos dificulta establecer la causa medicamentosa de esta entidad clínica.

Keywords: NET, SJS, Alergical expectorante, guaifenesina, diprofilina, Chlamydia, Mycoplasma, enterovirus.

Published November 9, 2017.

Copyright: (C) 2017 Author. This is an open-access article distributed under the terms of the Creative Commons Attribution License, which permits unrestricted use, distribution, and reproduction in any medium, provided the original author and source are credited. Editor: Alberto M. Borobia Pérez.

Cite as: Díaz García L., Rodríguez Mariblanca A., García García I., Cabañas Moreno MR., Martínez de Soto L., Ramírez García E. Sospecha de NET por Alergical expectorante en ausencia de datos histológicos y microbiológicos. IBJ Clin Pharmacol 2017 (S1):e0003 doi: $10.24217 / 2530-4984.17 v 1 s 1.00003$.

Funding: The authors declare no funding involved.

Competing Interests: The authors declare no conflicts of interest. 


\title{
Necrólisis epidérmica tóxica causada por Pilexil champú: serenoa serrulata vs hidantoína.
}

\author{
Rodríguez Mariblanca A, Díaz García L¹, García García I, Nanwani Nanwani K², Sendagorta E³, Ramírez García E.
}

${ }^{1}$ Servicio de Farmacología Clínica, Hospital Universitario La Paz, IdiPaz, Consorcio PIELenRed, Facultad de Medicina, Universidad Autónoma de Madrid, España ${ }^{2}$ Servicio de Cuidados Intensivos, Hospital Universitario La Paz, IdiPaz, Consorcio PIELenRed, Madrid, España.

${ }^{3}$ Servicio de Dermatología, Hospital Universitario La Paz, IdiPaz, Consorcio PIELenRed, Madrid, España.

*Corresponding author: Elena Ramírez. Servicio de Farmacología Clínica, Hospital Universitario La Paz, IdiPaz, Consorcio PIELenRed, Facultad de Medicina, Universidad Autónoma de Madrid, España, Dirección: Paseo de la Castellana 261. 28046, Madrid, España. Tel - fax: + 34 917277559; E-mail: elena.ramirez@inv.uam.es

Introducción: La necrolisis epidérmica tóxica (NET) es una reacción mucocutánea de causa medicamentosa en la mayoría de los casos asociada a una morbilidad y una mortalidad elevadas. Se produce una apoptosis generalizada de los queratinocitos y descamación de la piel, con erosiones en mucosas y síntomas sistémicos graves. El mecanismo exacto es desconocido, aunque se acepta que el mediador principal que conduce a la muerte de los queratinocitos es la granulisina. Lo más importante en el manejo de esta entidad es el diagnóstico precoz, la retirada de los medicamentos sospechosos y el tratamiento de soporte.

Material y métodos: Una paciente mujer de 58 años trasladada a Unidad de Quemados de HULP son sospecha de NET. En las pruebas complementarias no se objetivaron hallazgos que pudiesen justificar el cuadro excepto Mycoplasma IgG positiva (contacto pasado), por lo que se buscó una causa medicamentosa según el algoritmo ALDEN (Algorithm of drugs causality for EN).

Su tratamiento habitual era atorvastatina y levotiroxina desde hace más de 10 años y Pilexil cápsulas ${ }^{\circledR}$ y Pilexil champú $^{\circledR}$ desde 5 meses antes del comienzo del cuadro. Atorvastatina y levotiroxina, así como Pilexil cápsulas, presentaban una ventana de exposición improbable (> 56 días). Se realizó una revisión de la composición de Pilexil champú ${ }^{\circledR}$ ya que los productos tópicos podrían tener una lenta absorción y justificar una ventana de exposición prolongada.

Resultados: Entre la composición de Pilexil champú ${ }^{\circledR}$ se encontraron componentes activos: Serenoa Serrulata (palmetto, fitosteroles inhibidores de la $5 \alpha$-reductasa), tocoferil nicotinate (incrementa el flujo sanguíneo local), vitaminas (biotina, B6, vitamina E y provitamina B5) e DMDM hidantoína (conservante). Sus propiedades vasodilatadoras podrían favorecer la absorción de otros compuestos en pequeñas cantidades a lo largo de muchos meses, lo que finalmente podría haber desencadenado el cuadro. El palmetto es un extracto vegetal utilizado en el tratamiento de la hipertrofia benigna de próstata y en la alopecia androgénica, cuyos efectos adversos más conocidos son dermatitis, síntomas intestinales, cefalea, molestias urinarias, hemorragia cerebral, pancreatitis y hepatitis. Se ha descrito un caso de dermatitis por minoxidilo (vasodilatador periférico utilizado en el tratamiento de alopecia androgénica) con reactividad cruzada a Serenoa Serrulata en las pruebas cutáneas. La fenitoína, uno de los fármacos que con más frecuencia produce NET, es una hidantoína (difenilhidantoína).

Conclusiones: Puesto que hay descrito un caso de dermatitis por minoxidilo con reactividad cruzada a Serenoa Serrulata, no se puede descartar una relación química entre ellos. Por otro lado, es bien conocida la relación de la fenitoína con reacciones adversas cutáneas graves, por lo que también sería una sustancia sospechosa en este caso. Por tanto cualquiera de estas dos sustancias podrían ser la causa de la NET en esta paciente.

Keywords: necrólisis epidérmica tóxica, NET, Pilexil, serenoa serrulata, hidantoína.

Published November 9, 2017.

Copyright: (C) 2017 Author. This is an open-access article distributed under the terms of the Creative Commons Attribution License, which permits unrestricted use, distribution, and reproduction in any medium, provided the original author and source are credited. Editor: Alberto M. Borobia Pérez.

Cite as: Rodríguez Mariblanca A., Díaz García L., García García I., Nanwani Nanwani K., Sendagorta, E., Ramírez García E. Necrólisis epidérmica tóxica causada por Pilexil champú: serenoa serrulata vs hidantoína. IBJ Clin Pharmacol 2017 (S1):e0004 doi: 10.24217/2530-4984.17v1s1.00004.

Funding: The authors declare no funding involved.

Competing Interests: The authors declare no conflicts of interest. 


\title{
Hiperpigmentacion inducida por antihipertensivos.
}

\author{
Rosa Gimenez García ${ }^{1 *}$, Sergio Carrasco Molina ${ }^{1}$. \\ ${ }^{1}$ Servicio de Dermatología. Hospital Rio Hortega. Valladolid. España.

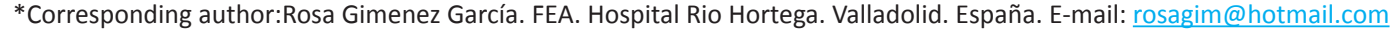

\begin{abstract}
Le ha estimado que entre un 10 al $20 \%$ de los casos de hiperpigimentacion adquirida se relaciona con un origen medicamentoso. Clásicamente se han descrito estas alteraciones inducidas por diferentes tipos de fármacos incluyendo los antimaláricos, amiodarona, citostáticos, tetraciclinas, metales pesados o psicofármacos. Se han publicado muy pocos casos de hiperpigmentacion inducida por fármacos utilizados en el tratamiento de la hipertensión arterial. Presentamos tres casos de hiperpigmentación inducida por antihipertensivos.
\end{abstract}

Caso 1. Paciente de 73 años, con antecedentes personales de alergia a la neomicina, que acude a la consulta de dermatología por presentar unas alteraciones de la pigmentación que se presentaron 4 meses después de iniciar un tratamiento con telmisartan asociado a hidroclorotiazida. A la exploración observamos lesiones hiperpigmentadas de diferentes tamaños, localizadas en los antebrazos, el dorso de los pies, en la región poplítea y en la semimucosa labial inferior. El estudio histológico puso de manifiesto una marcada incontinencia pigmenti. Tras la supresión del tratamiento las lesiones regresaron paulatinamente hasta su regresión a los pocos meses.

Caso 2. Se trata de un varon de 79 años de edad, con antecedentes personales de cáncer de próstata y de insuficiencia renal severa, que tras el comienzo de tratamiento antihipertensivo con telmisartan 80-hidroclorotiazida 125 refiere hiperpigmentación y prurito en las zonas expuestas al sol. Se había tratado con corticoides tópicos y diclofenaco sin mejoría. La exploración reveló la presencia de una pigmentacion gris pizarra en la cara, región del escote y el dorso de las manos. Tres meses después de cambiar de antihipertensivo prácticamente había desaparecido la pigmentación y el paciente estaba asintomático

Caso 3. Una mujer de 68 años de edad es derivada a nuestra consulta para la evaluación de unas lesiones faciales que aparecieron en verano tras exposición al sol y cuadro de eritema facial. En su historia personal destacaba una hipertensión arterial tratada con lecarnidipino en los últimos dos años. A la exploración objetivamos una pigmentacion reticulada gris parduzca localizada en los parpados, las sienes y las mejillas. La paciente ha mejorado muy lentamente, tras la discontinuación del fármaco y uso de fotoprotectores.

La hipertension constituye un problema de salud cada vez mas importante. En general, y a pesar de su uso muy frecuente, son muy raras del grupo las reacciones cutáneas inducidas por antihipertensivos ARA II o antagonistas del calcio. Hemos encontrado en la literatura algún caso de pigmentación inducida por captopril, diltiazem y amlodipino fundamentalmente. Las presentación clínica de las discromías de origen medicamentoso es muy variable con diferentes patrones de color, patrón y localización

Ante la aparición de lesiones hiperpigmentadas debe de sospecharse un origen medicamentoso, especialmente en ancianos tratados con antihipertensivos.

Keywords: hiperpigmentacion, antihipertensivos, telmisartan, larcanidipino.

Published November 9, 2017.

Copyright: (C) 2017 Author. This is an open-access article distributed under the terms of the Creative Commons Attribution License, which permits unrestricted use, distribution, and reproduction in any medium, provided the original author and source are credited. Editor: Alberto M. Borobia Pérez.

Cite as: Gimenez García R., Carrasco Molina S. Hiperpigmentacion inducida por antihipertensivos. IBJ Clin Pharmacol 2017 (S1):e0005 doi: 10.24217/2530-4984.17v1s1.00005.

Funding: The authors declare no funding involved.

Competing Interests: The authors declare no conflicts of interest. 


\title{
¿DRESS o Pustulosis? Esa es la cuestión.
}

\author{
Aroa García Duarte ${ }^{1}$, Alba Gómez Zubiaur ${ }^{1}$, Alicia Quesada Cortés ${ }^{1}$, lleana Medina Expósito ${ }^{2}$, Marta Bandini ${ }^{1}$, Isabel \\ Hernández Surmann ${ }^{1}$.
}

${ }^{1}$ Servicio de Dermatología. Hospital Universitario Príncipe de Asturias. Alcalá de Henares (Madrid).

${ }^{2}$ Servicio de Anatomía Patológica. Hospital Universitario Príncipe de Asturias. Alcalá de Henares (Madrid).

*Corresponding author: Aroa García Duarte. Hospital Universitario Príncipe de Asturias. Alcalá de Henares (Madrid); E-mail: aroagarciaduarte@ hotmail.com

Introducción: El síndrome de hipersensibilidad a fármacos con síntomas sistémicos y eosinofilia (DRESS) y la pustulosis exantemática generalizada aguda (PEGA) son dos reacciones de hipersensibilidad grave a fármacos. Si bien cada una de ellas presenta unas características clínicas y unos criterios diagnósticos definidos, en la práctica clínica habitual existen casos con características comunes.

Historia clínica: Mujer de 90 años con antecedentes de hipertensión arterial e insuficiencia cardíaca, que tras cuatro días de tratamiento con cefditorén y un día de tratamiento con levofloxacino por neumonía adquirida en la comunidad, presenta un exantema generalizado con pápulas eritemato-edematosas e infiltradas confluentes, algunas con dudosa morfología dianiforme, junto con lesiones purpúricas en abdomen y petequiales en miembros inferiores. Asocia fiebre de hasta 38,5ㅇ․ En la analítica destaca eosinofilia y linfopenia leve. Ante la sospecha de Síndrome de DRESS se realiza biopsia cutánea, se retiran los fármacos sospechosos y se inicia tratamiento con corticoterapia intravenosa. La biopsia mostró hallazgos compatibles con pustulosis exantemática generalizada aguda, con extensas pústulas subcórneas e intraepidérmicas. La paciente mostró una buena evolución clínica con resolución completa de las lesiones a lo largo de un mes con el tratamiento corticoideo.

Discusión: Presentamos un caso de toxicodermia grave clínicamente compatible con un síndrome de DRESS, en el que la anatomía patológica fue diagnóstica de PEGA. Revisando los criterios diagnósticos establecidos, la puntuación del RegiScar Score para Síndrome de DRESS fue de 4 (probable DRESS) y según el EuroScar Score para la PEGA de 4 (posible PEGA). Nos planteamos si se trata de un síndrome de solapamiento entre las dos entidades, de una PEGA con características comunes a un síndrome de DRESS, o bien son dos formas de reacción cutánea consecutiva. Han sido publicados síndromes de solapamiento entre DRESS y PEGA, tanto con eosinofilia como con neutrofilia en sangre periférica, y suponen un motivo de controversia, ya que hasta en el $20 \%$ de los casos de DRESS pueden observarse pústulas como manifestación cutánea. También puede haber casos que cumplan criterios de ambas entidades.

Conclusión: Nos planteamos la existencia de síndromes de solapamiento entre las toxicodermias graves y destacamos el papel de la biopsia para el diagnóstico de las mismas. La importancia del diagnóstico diferencial radica en las diferencias en cuanto a tratamiento y pronóstico de ambas entidades. Los corticoides sistémicos son la primera línea de tratamiento para el síndrome de DRESS, siendo eficaces como tratamiento de segunda línea las inmunoglobulinas intravenosas o la ciclosporina si aparecen efectos adversos o los corticoides son ineficaces. Sin embargo, la evolución de la PEGA es favorable tras la retirada del fármaco, con desaparición espontánea de las lesiones, siendo el tratamiento con corticoides controvertido.

Keywords: Toxicodermia, solapamiento, DRESS, PEGA.

Published November 9, 2017.

Copyright: (c) 2017 Author. This is an open-access article distributed under the terms of the Creative Commons Attribution License, which permits unrestricted use, distribution, and reproduction in any medium, provided the original author and source are credited. Editor: Alberto M. Borobia Pérez.

Cite as: García Duarte A., Gómez Zubiaur A., Quesada Cortés A., Medina Expósito I., Bandini M., Hernández Surmann I. ¿DRESS o Pustulosis? Esa es la cuestión. IBJ Clin Pharmacol 2017 (S1):e0006 doi: 10.24217/2530-4984.17v1s1.00006.

Funding: The authors declare no funding involved.

Competing Interests: The authors declare no conflicts of interest. 


\title{
Infección por Parvovirus B19 como imitadora de DRESS.
}

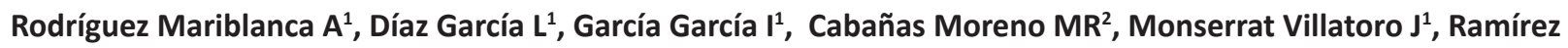 \\ García $\mathrm{E}^{1}$
}

${ }^{1}$ Servicio de Farmacología Clínica, Hospital Universitario La Paz, IdiPaz, Consorcio PIELenRed, Facultad de Medicina, Universidad Autónoma de Madrid, España ${ }^{2}$ Servicio de Alergia, Hospital Universitario La Paz, IdiPaz, Consorcio PIELenRed, Madrid, España.

*Corresponding author: Elena Ramírez. Servicio de Farmacología Clínica, Hospital Universitario La Paz, IdiPaz, Consorcio PIELenRed, Facultad de Medicina, Universidad Autónoma de Madrid, España, Dirección: Paseo de la Castellana 261. 28046, Madrid, España. Tel - fax: + 34 917277559; E-mail: elena.ramirez@inv.uam.es

Introducción: El síndrome DRESS es una reacción medicamentosa cutánea y sistémica que tiene múltiples complicaciones, un curso prolongado y una mortalidad de más del 10\%. Los signos característicos son fiebre, rash, eosinofilia, linfocitos atípicos, linfadenopatías, y hepatitis, así como reactivación de herpesvirus. Es un gran imitador de otras entidades (infecciones y enfermedades reumatológicas y hematológicas) entre ellas la infección por Parvovirus B19, cuyas manifestaciones clínicas son muy variadas. Se han descrito casos de infección por Parvovirus B19 que imitan a entidades como LES, infección por VEB, leucemia, VIH y DRESS.

Material y métodos: Una mujer de 45 años presentó un cuadro con rash eritematoso y pruriginoso en hombros y espalda que se generalizó, asociando edema facial, adenopatías laterocervicales y retroauriculares y febrícula de 37,7 으. En analítica elevación de AST (113UI/I) y eosinofilia $(700 \mathrm{~mm} 3)$. La paciente había realizado tratamiento con una dosis de fosfomicina $3 \mathrm{~g}$ (2a exposición) por ITU 3 días antes del inicio del cuadro e inició cefixima 2 días antes por PNA. Realizó tratamiento con Heliocare Ultra D durante las 3 semanas previas al inicio del cuadro. Fue diagnosticada de posible DRESS se recomendó no reexponer a fosfomicina y/o cefalosporinas y/o Heliocare ultra D, en espera de resultados de las serologías (Chlamydia, C. burnetii, CMV, Mycoplasma pneumoniae, VEB, VHS, VHB, VH6, Parvovirus B19) se inició tratamiento con prednisona 30mg en pauta descendente.

Resultados: Serologías negativas excepto Parvovirus B19 (IgG e IgM positivas). El curso de la enfermedad fue favorable, sin empeoramiento, y no requirió ingreso hospitalario. El hijo de 5 años de la paciente había presentado en el mes previo un cuadro de eritema generalizado con febrícula vespertina, astenia, hiporexia, heces acólicas, vómitos y pérdida de peso. En la analítica únicamente presentó eosinofilia. En serologías solicitadas por su pediatra posterior al diagnóstico de la paciente Parvovirus IgG positiva e IgM negativa (infección pasada). La infección por Parvovirus puede cursar con un amplio espectro de síntomas. En el $25 \%$ de los pacientes la infección se presenta con rash o eritema infeccioso y/o artralgias, como en este caso, aunque el eritema infeccioso suele ser más frecuente en niños. Estos síntomas son más frecuentes en la segunda semana tras el contagio, ya que en la primera semana la intensa viremia se acompaña de síntomas catarrales. En las infecciones agudas las pruebas serológicas muestran IgM positivo desde 7 a 10 días desde la exposición al virus.

Conclusiones: En nuestra paciente, la primoinfección está confirmada por las serologías. El curso de la enfermedad sin empeoramiento ni complicaciones no sería sugestivo de reactivación del Parvovirus por DRESS. El cuadro clínico similar en las dos entidades aportaría un argumento en favor del papel que juegan algunos virus en la aparición de estas manifestaciones clínicas en el DRESS.

Keywords: DRESS, Parvovirus B19, eritema, adenopatías.

Published November 9, 2017.

Copyright: (C 2017 Author. This is an open-access article distributed under the terms of the Creative Commons Attribution License, which permits unrestricted use, distribution, and reproduction in any medium, provided the original author and source are credited. Editor: Alberto M. Borobia Pérez.

Cite as: Rodríguez Mariblanca A., Díaz García L., García García I., Cabañas Moreno MR., Monserrat Villatoro J., Ramírez García E. Infección por Parvovirus B19 como imitadora de DRESS. IBJ Clin Pharmacol 2017 (S1):e0007 doi: 10.24217/25304984.17v1s1.00007.

Funding: The authors declare no funding involved.

Competing Interests: The authors declare no conflicts of interest. 


\title{
DRESS en paciente con toxoplasmosis y síndrome antisintetasa.
}

\author{
Marta Sánchez-Jareño ${ }^{1 *}$, Teresa Bellón ${ }^{2}$, Ana Fiandor ${ }^{1}$, Andreina Marques-Mejias ${ }^{1}$, Ana Entrala ${ }^{1}$, Rosario Cabañas ${ }^{1}$. \\ ${ }^{1}$ Servicio de Alergología, Hospital Universitario La Paz, IdiPAZ, Madrid. \\ ${ }^{2}$ Instituto de investigación sanitaria, Hospital Universitario La Paz, IdiPAZ, Madrid. \\ *Corresponding author: Marta Sánchez-Jareño. Servicio de Alergología, Hospital Universitario La Paz, Paseo de la Castellana 261, 28046, Madrid, \\ Spain. e-mail: martasanchezjare@gmail.com
}

Introducción: Se define como síndrome de DRESS al cuadro clínico grave caracterizado por la aparición de erupción cutánea, adenopatías, fiebre, eosinofilia, leucocitosis y/o afectación orgánica a las 2-6 semanas de iniciar tratamiento con un fármaco. Con mayor frecuencia, los fármacos implicados son anticonvulsivantes, sulfamidas, alopurinol y los antibióticos betalactámicos.

Historia clínica: mujer de 46 años con tratamiento inmunosupresor por síndrome antisintetasa que es diagnosticada de DRESS por cuadro de exantema con edema facial, fiebre ( $(\underline{a} 39,60 \mathrm{C})$, insuficiencia respiratoria e hipertransaminasemia con hepatoesplenomegalia y hemofagocitosis durante 11 días de tratamiento con sulfadiacina y pirimetamina para toxoplasmosis y 1 mes inicio de tratamiento con Diltiazem. Se traslada a la UCI con inicio de corticoterapia y mejoría. Se cambia tratamiento a sulfametoxazol/trimetoprim presentando nuevo exantema tras 30 días. Se instaura tratamiento con clindamicina/ pirimetamina reapareciendo a las 48 horas fiebre y exantema pruriginoso. Se decide suspender la medicación cediendo las lesiones en 10 días.

Resultados y discusión: Se realizaron los siguientes estudios analíticos donde se evidencia la elevación de las transaminasas con niveles máximos en tratamiento con sulfadiacina/pirimetamina y Diltiazem (AST $143 \mathrm{UI} / \mathrm{L}, \mathrm{ALT}$ 62UI/L, GGT 45 UI/L, Eo $200 \mathrm{cel} / \mathrm{mm} 3$ ), sulfametoxazol/trimetoprim (AST $406 \mathrm{UI} / \mathrm{L}$, ALT $306 \mathrm{UI} / \mathrm{L}, \mathrm{GGT} 562 \mathrm{UIL}$, Eo $440 \mathrm{cel} / \mathrm{mm} 3$ ) y clindamicina/ pirimetamina (AST 64 UI/L, ALT 49UI/L, GGT 170 UI/L, Eo 400). Serologías para virus hepatotropos negativas. Estudio alergológico a las 4 semanas de la resolución del cuadro: TTL positivo para: pirimetamina (IE 2,99), sulfadiacina (IE 22,75), sulfametoxazol $($ IE 9,64) y clindamicina (IE 2, 46). Negativo para Diltiazem (IE<2).

Conclusión: Presentamos un caso de síndrome DRESS probable según escala de Kardarun et al (4-5 puntos), inducido por sulfadiacina/pirimetamina con confirmación mediante TTL además de 2 reactivaciones posteriores, primero con sulfametoxazol/trimetoprim por reactividad cruzada entre sulfamidas y más tarde con clindamicina/pirimetamina igualmente con positividad en TTL.

Keywords: DRESS, TTL, SULFAMIDAS, TRIMETOPRIM, PIRIMETAMINA.

Published November 9, 2017.

Copyright: @ 2017 Author. This is an open-access article distributed under the terms of the Creative Commons Attribution License, which permits unrestricted use, distribution, and reproduction in any medium, provided the original author and source are credited. Editor: Alberto M. Borobia Pérez.

Cite as: Sánchez-Jareño M., Bellón T., Fiandor A., Marques-Mejias A., Entrala A., Cabañas R. DRESS en paciente con toxoplasmosis y síndrome antisintetasa. IBJ Clin Pharmacol 2017 (S1):e0008 doi: 10.24217/2530-4984.17v1s1.00008.

Funding: The authors declare no funding involved.

Competing Interests: The authors declare no conflicts of interest. 


\title{
Neosensibilización a betalactámicos durante síndrome de DRESS por fenitoína.
}

\author{
M. Andreína Marques-Mejías ${ }^{1 *}$, Marta Sánchez-Jareño ${ }^{1}$, Teresa Bellón ${ }^{2}$, Ana Fiandor ${ }^{1}$, Rosario Cabañas ${ }^{1}$.
}

${ }^{1}$ Servicio de Alergología. Hospital Universitario La Paz, Madrid, España.

${ }^{2}$ Instituto de Investigación sanitaria. Hospital Universitario La Paz-IdiPaz, Madrid, España.

${ }^{*}$ Corresponding author: M. Andreína Marques-Mejías. Servicio de Alergología. Hospital Universitario La Paz, Madrid, España. E-mail: mandreina. marques@gmail.com

Introducción: el síndrome de DRESS es una reacción severa y potencialmente mortal, poco frecuente. Se han descrito neosensibilizaciones a fármacos administrados a pacientes en la fase aguda del DRESS.

Caso clínico: varón de 55 años que en contexto de ictus inicia tratamiento con Fenitoína. Treinta y cinco días después presenta rash maculopapular generalizado, edema facial, fiebre, elevación de enzimas hepáticas y leucocitosis con eosinofilia. La clínica persistió más de 1 mes, cediendo progresivamente en tratamiento con corticoides sistémicos. Durante el ingreso se administró Cefepime en los primeros 15 días que se suspendió a los 10 días de tratamiento y se reintrodujo 1 mes después coincidiendo con empeoramiento de lesiones cutáneas y nuevo pico febril; se suspendió nuevamente durante 24 horas y se reintrodujo durante 5 días más. Así mismo se mantuvo tratamiento con Enoxaparina, Metamizol y Meropenem los últimos 20 días del ingreso, con buena tolerancia. Cuatro meses después se inicia tratamiento con Cefazolina y a los 3 días comienza a presentar exantema morbiliforme generalizado y febrícula, sin alteraciones analíticas. Se aumenta dosis de corticoides y a los 3 días ceden las lesiones. Desde el alta se suspenden Betalactámicos y Metamizol. Se realiza Test de transformación linfocitaria (TTL) 6 meses posterior al alta, que es claramente positivo con Fenitoína y negativo para Metamizol y Enoxaparina. Un año después el paciente acude a revisión para completar estudio de Betalactámicos: se realizan pruebas de intradermorreacción resultando positivas para PPL y MD en lectura inmediata. Positivas a las 24 y 48 horas para Cefalosporinas (Cefepime, Cefazolina y Ceftriaxona). Se realiza nuevo TTL que es positivo para Penicilina G, Cefazolina, Cefepime, Meropenem y Fenitoína. Se diagnostica al paciente de DRESS por fenitoína y neosensibilización a beta-lactámicos.

Discusión: se han descrito pocos casos de similares características en la literatura, la mayor serie fue descrita en España por Pere Gaig y col. En estos pacientes se descarta la posibilidad de reactividad cruzada entre fármacos, por la falta de similitud estructural. Podría estar en relación con la exposición a ciertas drogas en un período de depresión inmunológica, como es el primer episodio de DRESS. Este síndrome podría causar un estado de activación inmunitaria masiva, no especifica, que se traduce en una mayor expresión de moléculas de co-estimulación y citoquinas proinflamatorias. Conllevado a su vez, a una presentación antigénica más eficiente y reduciendo la tolerancia a otras drogas, como podrían ser los betalactámicos.

Conclusión: debe evitarse la administración de fármacos de forma empírica en DRESS por el riesgo de desarrollo de neosensibilizaciones.

Keywords: DRESS, SHA, neosensibilización, Test de activación linfocitaria.

Published November 9, 2017.

Copyright: (C) 2017 Author. This is an open-access article distributed under the terms of the Creative Commons Attribution License, which permits unrestricted use, distribution, and reproduction in any medium, provided the original author and source are credited. Editor: Alberto M. Borobia Pérez.

Cite as: Marques-Mejías MA., Sánchez-Jareño M., Bellón T., Fiandor A., Cabañas R. Neosensibilización a betalactámicos durante síndrome de DRESS por fenitoína. IBJ Clin Pharmacol 2017 (S1):e0009 doi: 10.24217/2530-4984.17v1s1.00009.

Funding: The authors declare no funding involved.

Competing Interests: The authors declare no conflicts of interest. 